\title{
Tin inventory for HVM EUVL sources
}

\author{
Martin C. Richardson, Kazutoshi Takenoshita, and Tobias Schmid \\ College of Optics \& Photonics, University of Central Florida, \\ 4000 Central Florida Blvd., Orlando, Florida, 32816-2700.
}

\begin{abstract}
Tin is one of the most efficient source materials for both gas discharge plasma sources and laser produced plasma sources for EUV lithography. Unlike Xenon which was the material commonly investigated for the EUVL source application, recycling of the target materials is not necessary for tin targets because of its low relative cost. However, in assessing the benefits of different source architectures, there are large differences in the size of the tin inventory used, and consequences that ensue. In this paper we make a first attempt to compare these differences, and assess their impact. Utilizing tin as the radiator at $13.5 \mathrm{~nm}$ reduces the total cost of the source system significantly.
\end{abstract}

Keywords: EUV sources, Droplet plasma, debris, inventory

\section{INTRODUCTION}

The choice of the target materials for powerful EUVL sources has been determined primarily based on the socalled in-band-conversion efficiency (CE) of the EUV radiation energy output over the energy input to create the source plasma. Tin is one of the most efficient materials for both gas discharge plasma sources and laser produced plasma sources. ${ }^{1-3}$ Unlike Xenon which was the material commonly investigated for the EUVL source application, recycling of the target materials is not necessary for tin targets because of the cost of the material itself. However, in assessing the benefits of different source architectures, there are large differences in the size of the tin inventory used, and consequences that ensue. For the long term source operation, to satisfy the $>30,000$ hours operation required, ${ }^{4}$ the target material inventory for a tin plasma source implementation can for some source architectures be significantly large. For these sources therefore, recycling of tin in the source system will indeed need to be considered. Here we make an assessment of the tin inventory required for most of the current source architectures, making reasonable assumptions on source and target operations for a source that satisfies roadmap requirements. The inventory of tin used in a high power EUV source used together with a high throughput stepper may well have strong impact on the cost-of-ownership (COO), and on the technical operation of the source. If this is the case, then our own design for an efficient EUV source, tin-doped droplet laser plasma source may have some advantages. This is a mass-limited $\operatorname{target}^{8}$ that minimizes not only the debris from the plasma but also the total amount of tin used. The number of tin atoms in a single droplet is of the order of $10^{13}$. This limited amount of tin contained in a single target requires much less tin inventory than other source architectures, such as tin discharge plasma sources ${ }^{2,9,10}$ and tin droplet laser plasma sources. ${ }^{11,12}$ In this paper we make a first attempt to compare the tin inventory for those different source architectures and assess their impact.

Further author information:

Kazutoshi Takenoshita: E-mail: ktakenos@creol.ucf.edu, Telephone: 14078236800 


\section{SIMPLE MODEL OF TIN MATERIAL USAGE}

Our primary purpose in this paper is gain a quantitative assessment of the total amount of tin needed for each source, assuming that it satisfies the current roadmap EUV requirements. We address less the technical and operational consequences of the inventories we estimate, beyond making some obvious comments with respect to operation and configuration. We first construct a simple model that takes into account the mass of tin used in each source architecture, the manner of its operation, and the need, if necessary, for recycling.

\subsection{Basic expressions of the amount of tin}

There are two practical aspects that affect the operation of all EUVL source systems over a long time. Firstly we assess the total amount of tin inventory needed for the system to run 30,000 hours without recourse to recycling the tin used. Then we determine the impact of tin recycling taking into account that fraction of the tin used directly in the generation of EUV radiation, and that fraction that is needed for the source to function, but does not directly contribute to the EUV radiation. It is important to consider these two aspects for the different source architectures and to make a reasonable balance of the cost of the inventory and the cost of the recycling. Therefore the model used for calculating the tin inventory in this paper covers both the total tin inventory and the amount of tin required with constant recycling.

The simplest way to calculate the total tin inventory for a EUVL source system is to multiply the amount of tin supplied in a single plasma generation and the total number of plasma generated in the entire lifetime, which is expressed as,

$$
M_{\text {total }}=M_{\text {target }} \times f_{\text {source }} \times T_{\text {lifetime }}
$$

where $M_{\text {total }}$ is the total mass of tin in $\mathrm{kg}$ which would be used in a single source unit, $M_{\text {target }}$ is the mass of tin consumed for producing a single EUV plasma pulse, $f_{\text {source }}$ is the frequency of the source plasma in $\mathrm{Hz}$, and $T_{\text {lifetime }}$ is the total lifetime of the source system in seconds. Here we assumed that $T_{\text {lifetime }}$ would be the 30,000 hour $\left(1.1 \times 10^{8} \mathrm{~s}\right)$ lifetime currently specified, based on the required source cleanliness.

By using Eqn. 1 it is possible to illustrate the impact of the tin inventory required for the different source configurations. However, for a more precise model of the tin usage, we calculate the tin inventory required for different periods. We define $M_{\text {sup }}$ as the mass of the tin target material supply for a specific time period. This is obtained from the unit tin content $m_{\text {sup }}$ and the frequency of the target supply $f_{\text {sup }}$ for most of the configurations. Thus,

$$
M_{\text {sup }}=m_{\text {sup }} \times f_{\text {sup }} \times T_{\text {sup }}
$$

where $m_{\text {sup }}$ in $\mathrm{kg}, f_{\text {sup }}$ in $\mathrm{Hz}$, and $T_{\text {sup }}$ in seconds. For droplet tin supply cases $m_{\text {sup }}$ is defined as the mass of tin contained in a single droplet target. For discharge plasma source configurations utilizing tin-coated electrode, $m_{\text {sup }}$ is defined as the mass of tin ablated by each laser pulse to generate the tin gas atmosphere required for the discharge source. For sources that use continuous tin jet, $m_{\text {sup }}$ is defined as the mass tin supplied in a period of each source plasma generation.

We now relate this value of the mass of tin supplied, $m_{\text {sup }}$, to (i) the mass of tin, $m_{\text {rad }}$ (in $\mathrm{kg}$ ), used to produce the EUV radiation for each plasma generation, (ii) the mass of tin $m_{r e t}$, retrieved, and the mass of tin lost as waste $m_{\text {was }}$. Thus,

$$
m_{\text {sup }}=m_{\text {rad }}+m_{\text {ret }}+m_{\text {was }}
$$

Ideally we would like the mass of tin retrieved, $m_{\text {ret }}$, and the mass wasted, $m_{\text {was }}$, to be zero. This is our definition of a mass-limited target for a laser plasma source. ${ }^{8}$ Obviously if a larger amount tin needs to be used in a given source, the mass of tin wasted, $m_{\text {was }}$, should be minimized. 


\subsection{Total amount of tin in On-Demand target supply schemes}

To predict the amount of tin used in an on-demand based target source, the equations must be modified to include the difference between the wafer exposure time and the total dwell time for the wafer in the stepper (including incoming transport, registration, exposure and outgoing transport from the stepper). In this case, the tin supply must be precisely controlled to correspond to the required plasma generation. Therefore the total mass of tin supplied in a specified operation period is expressed as

$$
M_{\text {sup }}=m_{\text {sup }} \times f_{\text {sup }} \times \frac{T_{\text {exp }}}{T_{w a f}} \times T_{\text {opr }}
$$

where $T_{\text {exp }}$ is the wafer exposure time, $T_{\text {waf }}$ is the dwell time for a wafer, and $T_{o p r}$ is the operation time period. Previous models for an EUV stepper have used $T_{\exp }=9 \mathrm{~s}$ and $T_{w a f}=36 \mathrm{~s}$ for a throughput of 100 wafers $/ \mathrm{hr}$ with the assumption of a resist sensitivity of $5 \mathrm{~mJ} / \mathrm{cm}^{2} .{ }^{13}$ All the calculated values in the next section refer to the values.

A similar expressions can be derived for the mass of tin used in generating useful EUV radiation, $M_{\text {rad }}$, expressed as,

$$
M_{\text {rad }}=m_{\text {rad }} \times f_{\text {pla }} \times \frac{T_{e x p}}{T_{w a f}} \times T_{o p r}
$$

where $f_{p l a}$ is the frequency of the source plasma generation in Hz. Likewise we can create similar expressions for the mass of tin retrieved from recycling, $M_{r e t}$, and the mass of tin wasted, $M_{\text {was }}$,

$$
\begin{gathered}
M_{r e t}=m_{r e t} \times f_{p l a} \times \frac{T_{e x p}}{T_{w a f}} \times T_{o p r}, \\
M_{w a s}=m_{w a s} \times f_{p l a} \times \frac{T_{e x p}}{T_{w a f}} \times T_{o p r} .
\end{gathered}
$$

Thus there is relationship for the tin mass supply for an on-demand target supply scheme, similar to that in Eqn. 3, expressed,

$$
M_{\text {sup }}=M_{\text {rad }}+M_{\text {ret }}+M_{\text {was }} .
$$

For an on-demand target system, we would ideally prefer the amount of tin wasted to be minimized. This can be achieved in a trade-off between the size and frequency of the droplet generation and the laser frequency. Including these factors would modify the expressions above.

\subsection{The general form of the mass of tin retrieved}

For other sources, particularly those that use continuous streams of droplets at frequencies higher than the laser or electrical drive frequencies, or for the case of continuous jet, the Eqn. 6 is no longer valid, since some fraction of the source supply is not used in source plasma generation. In this case, a simplified assumption would be that these target are retrieved without any mass reduction.

The general form of the mass of tin retrieved is expressed as,

$$
\begin{aligned}
M_{\text {ret }}= & m_{\text {ret }} \times f_{\text {pla }} \times \frac{T_{\text {exp }}}{T_{\text {waf }}} \times T_{\text {opr }} \\
& +m_{\text {sup }} \times\left(f_{\text {sup }}-f_{\text {pla }}\right) \times \frac{T_{\text {exp }}}{T_{\text {waf }}} \times T_{\text {opr }} \\
& +m_{\text {sup }} \times f_{\text {sup }} \times \frac{T_{\text {waf }}-T_{\text {exp }}}{T_{\text {waf }}} \times T_{\text {opr }}
\end{aligned}
$$

where the first term of right hand side is the same expression as described in On-Demand target supply scheme, the second term is the amount of tin target supplied at higher frequency than the plasma frequency, and the 
third term is the target supplied during wafer loading and unloading. This third term is necessary for all schemes with a continuous target supply. Depending on the amount of tin retrieved expressed in Eqn. 9, tin recycling schemes may be necessary.

These expressions will be applied to different source architectures in the next section to illustrate the impact to the tin inventory. The amount of tin retrieved $M_{\text {ret }}$ will also illustrate how important or necessary tin recycling schemes are in different source architectures. The tin inventory required for any periods of operation is indicated by $M_{\text {sup }}$. The tin material consumption is the sum of $M_{\text {rad }}$ and $M_{\text {was }}$. Most importantly the amount of tin wasted $M_{\text {was }}$ impacts the entire source system since the wasted tin will spread all over the surface of the source chamber. Any debris mitigation schemes installed in the source chamber must be capable of accepting a large fraction of the wasted amount.

\section{CASE STUDIES}

Here we describe four different source architectures, (i) the tin-doped droplet target supply, (ii) the discharge source with tin-coated electrodes, (iii) the tin-droplet target supply, and (iv) the discharge source with tin-jet electrodes. Each source architecture is modeled by specifying the unit mass of tin, $m_{\text {sup }}$, the mass of tin used to produce EUV radiation, $m_{\text {rad }}$, the mass of tin retrieved from a plasma generation, $m_{r e t}$, and the tin wasted in oversupplying the target material, $m_{\text {was }}$. The tin inventory required and tin consumption for two operation periods are calculated by the model described above. The tin inventory estimated for a week operation helps assess whether or not recycling tin is required. In contrast, the tin consumption for a year operation can indicate the impact on a source chamber and debris mitigation schemes from the mass of tin wasted and accumulated over the period.

\subsection{Tin-doped droplet plasma source}

We have proposed the tin-doped droplet target for EUVL sources for many years. The target supply scheme is capable of long term operation because of its high $\mathrm{CE}$, low debris, and its low mass of tin content in a single droplet. As mentioned previously, $m_{r e t}$ and $m_{\text {was }}$ for tin-doped droplet target scheme are effectively zero. This property is one of its prime advantages over other target/source architectures.

The mass of tin contained in a single droplet target is typically $2.0 \times 10^{-12} \mathrm{~kg}$ and this value is the unit mass of tin $m_{\text {sup }}$ for this target system. For EUV power generation for HVM, the source plasma will be generated at a frequency of higher than $30 \mathrm{kHz}$. Here the source frequency, $f_{\text {pla }}$, is set to be $30 \mathrm{kHz}$. When the source repetition rate needs to be higher, the calculations of the mass of tin will be scaled accordingly. To illustrate the capability of high repetition rate of the source plasma, $f_{\text {sup }}$ is set to be $100 \mathrm{kHz}$. All the target parameters are summarized in the Table 1.

Table 1. Parameters for tin-doped droplet target scheme.

$\begin{array}{lll}\text { Mass of tin supplied [kg] } & \left(m_{\text {sup }}\right) & 2.0 \times 10^{-12} \\ \text { Mass of tin used in EUV generation }[\mathrm{kg}] & \left(m_{\text {rad }}\right) & 2.0 \times 10^{-12} \\ \text { Mass of tin retrieved [kg] } & \left(m_{\text {ret }}\right) & 0 \\ \text { Mass of tin wasted }[\mathrm{kg}] & \left(m_{\text {was }}\right) & 0 \\ \text { Rep. rate of target supply }[\mathrm{kHz}] & \left(f_{\text {sup }}\right) & 100 \\ \text { Rep. rate of source operation }[\mathrm{kHz}] & \left(f_{\text {pla }}\right) & 30\end{array}$

The mass of tin supplied, used to produce EUV, retrieved, and wasted are calculated by using Eqns. 2, 3, 7 , and 9. The calculations do not include any down time for the EUV source operation. The tin material usage is summarized in Table 2. Tin consumption for this scheme is only $9 \mathrm{~g}$ per week or $470 \mathrm{~g}$ per year. It is limited to 
Table 2. Total mass of tin for tin-doped droplet target scheme

\begin{tabular}{lr|r|r} 
Tin usage & & per week $[\mathrm{kg}]$ & per year $[\mathrm{kg}]$ \\
\hline Mass of tin supplied & $\left(M_{\text {sup }}\right)$ & 0.12 & 6.24 \\
Mass of tin used in EUV generation & $\left(M_{\text {rad }}\right)$ & 0.009 & 0.47 \\
Mass of tin retrieved & $\left(M_{\text {ret }}\right)$ & 0.11 & 5.77 \\
Mass of tin wasted & $\left(M_{\text {was }}\right)$ & 0 & 0
\end{tabular}

the mass of tin used in generating EUV radiation. This scheme only requires tin inventory of only $\sim 120 \mathrm{~g}$ for a week operation, and $\sim 6 \mathrm{~kg}$ for a year operation. The mass of tin retrieved for a week operation is only $\sim 110$ $\mathrm{g}$ and $\sim 6 \mathrm{~kg}$ for a year operation. This small amount of tin retrieved can easily be handled without recycling of tin.

\subsection{Discharge plasma sources with tin-coated electrodes}

The tin material required for discharge sources using liquid tin electrodes is governed by mainly by volume of tin required for a smooth electrode, and by the ablation of the tin coated electrode by the focused laser beam. ${ }^{2,9}$ The mass of tin required can be varied by many orders of magnitude. However, here we make reasonable assumptions using the expressions described in the previous section.

The mass of tin supplied should be equal to the mass of tin removed from the electrode by a laser pulse. For a crater made by a laser beam, the volume of tin ablated $V_{a b l}$ can be approximated by

$$
V_{a b l}=\pi \times\left(\frac{D}{2}\right)^{2} \times d
$$

where $D$ is the diameter of the crater, and $d$ is the averaged depth of the crater. We conservatively estimate these to be $80 \mu \mathrm{m}$ diameter and $25 \mu \mathrm{m}$ deep respectively, giving the ablated volume as $1.3 \times 10^{-7} \mathrm{~cm}^{3}$. With the density of tin, the mass of tin supplied to a plasma is $9.5 \times 10^{-10} \mathrm{~kg}$. The mass of tin used to produce EUV is considered to be 3 times more than the tin-doped droplet target case because of the 3 times lower the plasma frequency in discharge plasma sources. The mass of tin retrieved can be about $30 \%$ of the supplied mass, which is an approximated ratio of solid angle of the electrodes intercepting of isotropic tin material emission. These recaptured tin material by electrodes can be reuse for later plasma generation. From Eqn. 3, the mass of tin wasted is calculated. All the target parameters are shown in Table 3 and the mass of tin usage for a week and a year are shown in Table 4. Here the mass of tin used in coating the electrodes is not included in the calculations since the electrode surface area depends on the electrode geometry. We would also expect that the effects of laser-plasma-generated shock waves propagating through the liquid tin surface, would give rise to hydrostatic turbulent effects that might splatter additional tin within the source chamber.

Table 3. Parameters for discharge plasmas with tin-coated electrodes.

$\begin{array}{lll}\text { Mass of tin supplied [kg] } & \left(m_{\text {sup }}\right) & 9.5 \times 10^{-10} \\ \text { Mass of tin used in EUV generation }[\mathrm{kg}] & \left(m_{\text {rad }}\right) & 6.0 \times 10^{-12} \\ \text { Mass of tin retrieved [kg] } & \left(m_{\text {ret }}\right) & 2.9 \times 10^{-10} \\ \text { Mass of tin wasted [kg] } & \left(m_{\text {was }}\right) & 6.6 \times 10^{-10} \\ \text { Rep. rate of target supply [kHz] } & \left(f_{\text {sup }}\right) & 10 \\ \text { Rep. rate of source operation }[\mathrm{kHz}] & \left(f_{\text {pla }}\right) & 10\end{array}$

The tin consumption for this source is large. It requires several kilograms of tin each week of operation. The important aspect of the tin material usage in this source architecture is the mass of tin wasted. Only a small 
Table 4. Total mass of tin for discharge plasmas with tin-coated electrodes.

\begin{tabular}{lr|r|r} 
Tin usage & & per week $[\mathrm{kg}]$ & per year $[\mathrm{kg}]$ \\
\hline Mass of tin supplied & $\left(M_{\text {sup }}\right)$ & 1.43 & 74.1 \\
Mass of tin used in EUV generation & $\left(M_{\text {rad }}\right)$ & 0.009 & 0.47 \\
Mass of tin retrieved & $\left(M_{\text {ret }}\right)$ & 0.43 & 22.2 \\
Mass of tin wasted & $\left(M_{\text {was }}\right)$ & 0.99 & 51.4
\end{tabular}

part of tin ablated on the electrode surface actually contributes to generating useful EUV radiation. Most of ablated tin is spread throughout the source chamber, in particularly towards the debris mitigation system. The mass of tin which will be coated on the surface of the mitigation schemes is $\sim 1 \mathrm{~kg}$ for a week operation and $\sim 50$ $\mathrm{kg}$ for a year operation. Even if additional recycling schemes are installed on the mitigation schemes, handling this large amount of tin may be a challenge.

\subsection{Tin droplet laser plasma sources}

Solid tin droplet target architectures are being proposed not only for laser plasma sources but also discharge plasma sources..$^{9,11,12}$ This target is distinguished from the tin-doped droplet target, in that it only contains only tin at solid/liquid density. The droplet delivery, at the plasma frequency or higher, is facilitated either by an On-Demand or by a continuous delivery system. Here we estimate the most conservative use of tin by considering the tin consumption for an On-Demand target delivery where the frequency of the droplet generation is higher than the plasma frequency.

All the target parameters are shown in Table 5 where the diameter of tin droplet is $52 \mu \mathrm{m}$ at the droplet frequency of $48 \mathrm{kHz}$. It is assumed that the tin flow rate is same as that for the previously reported droplet diameter of $100 \mu \mathrm{m}$ at $7 \mathrm{kHz} .^{3}$ The mass of tin used in radiation is set to the equivalent mass for tin-doped droplet target case. The mass of tin retrieved from the target being shot by laser is $50 \%$ of a whole droplet. The plasma frequency is taken from the number presented previously. ${ }^{11}$

Table 5. Parameters for tin droplet target architectures.

$\begin{array}{lll}\text { Mass of tin supplied }[\mathrm{kg}] & \left(m_{\text {sup }}\right) & 5.6 \times 10^{-10} \\ \text { Mass of tin used in EUV generation }[\mathrm{kg}] & \left(m_{\text {rad }}\right) & 2.5 \times 10^{-12} \\ \text { Mass of tin retrieved [kg] } & \left(m_{\text {ret }}\right) & 2.8 \times 10^{-10} \\ \text { Mass of tin wasted }[\mathrm{kg}] & \left(m_{\text {was }}\right) & 2.8 \times 10^{-10} \\ \text { Rep. rate of target supply }[\mathrm{kHz}] & \left(f_{\text {sup }}\right) & 48 \\ \text { Rep. rate of source operation }[\mathrm{kHz}] & \left(f_{\text {pla }}\right) & 24\end{array}$

Table 6. Total mass of tin for tin droplet target architectures.

\begin{tabular}{lc|r|r} 
Tin usage & & per week $[\mathrm{kg}]$ & per year $[\mathrm{kg}]$ \\
\hline Mass of tin supplied & $\left(M_{\text {sup }}\right)$ & 4.0 & 210 \\
Mass of tin used in EUV generation & $\left(M_{\text {rad }}\right)$ & 0.009 & 0.47 \\
Mass of tin retrieved & $\left(M_{\text {ret }}\right)$ & 3.0 & 157 \\
Mass of tin wasted & $\left(M_{\text {was }}\right)$ & 1.0 & 52
\end{tabular}

The total usage of tin for the tin droplet target architecture is summarized in Table 6 using Eqns. 4, 5, 7, and 9 . The tin inventory required for this source configuration is at least $4 \mathrm{~kg}$ for a week operation assuming at 
least $3 \mathrm{~kg}$ of tin must be retrieved. Tin consumption is $1 \mathrm{~kg}$ per week and more than $50 \mathrm{~kg} /$ year. The consumed tin will be distributed into the source chamber and debris mitigation schemes. The total mass of tin handling is more than $200 \mathrm{~kg}$ for a year while the mass of tin producing useful EUV radiation is $<1 \mathrm{~kg} /$ year.

\subsection{Discharge plasma sources with tin-jet electrodes}

We have also used the model described above to assess tin usage in discharge that use a liquid tin-jet electrode. ${ }^{10}$ The tin jet geometry is assumed to have a diameter of $100 \mu \mathrm{m}$ and the distance from the plasma position to the exits of the jets is assumed to be $10 \mathrm{~mm}$ in order to provide small erosion of the surface of the jet generator. We assume a jet velocity of $10 \mathrm{~m} / \mathrm{s}$ for $1 \mathrm{~mm}$ jet travel between plasmas operated at $10 \mathrm{kHz}$ so that the electrode geometry recovers after each plasma generation. The resistance of each electrode is $0.63 \Omega$ with the resistivity of $50 \times 10^{-8} \Omega \cdot \mathrm{m}$ at $300^{\circ} \mathrm{C}$. The joule heat generated in the tin-jet must be minimized to have an efficient source. A larger diameter of the jet provides lower loss in the tin-jet electrode but the mass of tin will be increased. On the other hand, shorter electrodes provide lower loss but the tin-jet generator will suffer high heat flux and energetic ion flux from the source plasma.

We assume the following for modeling this target. The tin-jets are assumed to be supplied on-demand during the wafer exposure time. The mass of tin is assumed to be equal to that in the discharge sources utilizing tincoated electrodes. The mass of tin used to produce EUV is also assumed to be the same as that in the discharge sources. A large fraction of the tin jet material is undisturbed by the plasma and only $\sim 10 \%$ of the mass of tin ablated is assumed to be intercepted and recaptured by the jets. The source parameters are shown in Table 7 .

Table 7. Parameters for discharge with tin-jet electrodes.

$\begin{array}{lll}\text { Mass of tin supplied }[\mathrm{kg}] & \left(m_{\text {sup }}\right) & 1.1 \times 10^{-7} \\ \text { Mass of tin used in EUV generation }[\mathrm{kg}] & \left(m_{\text {rad }}\right) & 6.0 \times 10^{-12} \\ \text { Mass of tin retrieved }[\mathrm{kg}] & \left(m_{\text {ret }}\right) & 1.0915 \times 10^{-7} \\ \text { Mass of tin wasted }[\mathrm{kg}] & \left(m_{\text {was }}\right) & 8.5 \times 10^{-10} \\ \text { Rep. rate of source operation }[\mathrm{kHz}] & \left(f_{\text {pla }}\right) & 10\end{array}$

Table 8. Total mass of tin for discharge with tin-jet electrodes.

\begin{tabular}{lr|r|r} 
Tin usage & & per week $[\mathrm{kg}]$ & per year $[\mathrm{kg}]$ \\
\hline Mass of tin supplied & $\left(M_{\text {sup }}\right)$ & $(165)$ & $(8580)$ \\
Mass of tin used in EUV generation & $\left(M_{\text {rad }}\right)$ & 0.009 & 0.47 \\
Mass of tin retrieved & $\left(M_{\text {ret }}\right)$ & $(164)$ & $(8506)$ \\
Mass of tin wasted & $\left(M_{\text {was }}\right)$ & 1.27 & 66
\end{tabular}

The tin usage is summarized in Table 8. The mass of tin supplied exceeds $100 \mathrm{~kg}$ for even a week operation. This indicates clearly there is a need for tin recycling system for this architecture. Even if a recycling system is installed, the mass of tin wasted exceeds $1 \mathrm{~kg}$ for a week's operation and $\sim 60 \mathrm{~kg}$ for a year's operation.

\section{CONCLUSION}

The tin inventory required to operate different source architectures is assessed. We compare the tin use and consumption for four different Tin-based EUV sources, assuming they each satisfy HVM power requirements. The use of our tin-doped droplet target scheme considerably reduces the tin inventory required for a long term 
operation, even without tin recycling. The main reason for the small inventory required for this source architecture is its use as a mass-limited target, in which no tin material is wasted in the plasma generation. In contrast the solid tin droplet source architecture and other discharge source architectures waste large amounts of tin. This large tin consumption will increase the tin inventory even for a short-term operation. The mass of tin used in generating EUV radiation is very small fraction of the mass of tin supplied to the plasma. Retrieval and recycling of the excess materials is therefore key for these high tin-consuming source architectures, otherwise several kilograms of tin will be deposited onto the debris mitigation schemes each week of operation. This will be more than $50 \mathrm{~kg}$ after a year.

\section{ACKNOWLEDGMENTS}

The authors acknowledge all the support from the members in Laser Plasma Laboratory, College of Optics \& Photonics, University of Central Florida. This work is supported by Powerlase Ltd., SRC, Intel, AMD, Cymer, and the State of Florida.

\section{REFERENCES}

1. C-S Koay, S. George, K. Takenoshita, R. Bernath, E. Fujiwara, M. Richardson, and V. Bakshi, "High conversion efficiency microscopic tin-doped droplet target laser-plasma source for EUVL," proceedings of SPIE 5751, pp. 279-292, 2005.

2. J. Pankert, et. al., "Integrating Philips' extreme UV source in the alpha-tools," proceedings of SPIE 5751, pp. 260-271, 2005.

3. D. W. Myers, I. V. Fomenkov, B. A. M. Hansson, B. C. Klene, and D. C. Brandt, "EUV source system development update: advancing along the path to HVM," proceedings of SPIE 5751, pp. 248-259, 2005.

4. A. Miyake, "Joint Requirements," presentation at EUV Source workshop, Oct. 19 2006, Barcelona, Spain, proceedings is avaiable at www.sematech.org.

5. S. A. George, C-S Koay, K. Takenoshita, R. Bernath, M. Al-Rabban, C. Keyser, V. Bakshi, H. Scott, M. Richardson, "EUV spectroscopy of mass-limited Sn-doped laser micro-plasmas," Proceedings of SPIE 5751, pp. $779-788,2005$

6. K. Takenoshita, C-S Koay, S. Teerawattanasook, M. Richardson, and V. Bakshi, "Debris characterization and mitigation from microscopic laser-plasma tin-doped droplet EUV sources," proceedings of SPIE $\mathbf{5 7 5 1}$, pp. 563-571, 2005.

7. M. Richardson, presentation at EUV Source Workshop, Oct. 19, 2006, Barcelona, Spain, proceedings is avaiable at www.sematech.org.

8. F. Jin, K. Gabel, M. C. Richardson, M. Kado, A. Vassiliev, D. Salzmann, "Mass-limited laser plasma cryogenic target for $13 \mathrm{~nm}$ point x-ray sources for lithography," Proc. SPIE 2015, pp. 151-159 1994.

9. U. Stamm, "High power EUV source development for beta-level and high volume manufacturing lithography," presentation at EUVL symposium, Oct. 16, Barcelona, Spain, proceedings is avaiable at www.sematech.org.

10. B. Vadime, H-J Voorma, et. al., "Requirements and prospects of next generation Extreme Ultraviolet Source for Lithography Applications," presentation at EUVL symposium, Oct. 16, Barcelona, Spain, proceedings is avaiable at www.sematech.org.

11. D. Brandt, "LPP EUV Source Development for HVM," Presentation at EUVL symposium, Oct. 17 2006, Barcelona, Spain, proceedings is avaiable at www.sematech.org.

12. A. Endo, "Development status of HVM Laser Produced Plasma EUV Light Source," Presentation at EUVL symposium, Oct. 17 2006, Barcelona, Spain, proceedings is avaiable at www.sematech.org.

13. K. Ota, Y. Watanabe, V. Banine, and H. Franken, "EUV Source Requirements for EUV Lithography," ed. V. Bakshi, EUV Sources for Lithography, SPIE, Bellingham, 2006. 